\title{
A Simple Transflective LCD for Mobile Applications
}

\author{
Yan Li, Zhibing Ge, and Shin-Tson Wu, Fellow, IEEE
}

\begin{abstract}
A double-cell-gap transflective liquid crystal display (TR-LCD) based on $63.6^{\circ}$ twisted nematic mode is proposed. With only one uniaxial positive A-film in transmissive region, this normally black TR-LCD exhibits several attractive features, such as high optical efficiency, low operation voltage, acceptable viewing angle, and good cell gap tolerance. Moreover, the electro-optical characteristics of reflective and transmissive regions match with each other well. Its fabrication process is fairly simple and it is suitable for mobile applications.
\end{abstract}

Index Terms-Double cell gap, high contrast ratio, positive A-film, transflective liquid crystal display (TR-LCD).

\section{INTRODUCTION}

$\mathbf{T}$ RANSFLECTIVE liquid crystal displays (TR-LCDs) have been widely used in mobile devices because of their good sunlight readability. To balance the optical path disparity between transmissive (T) and reflective (R) regions, double-cell-gap method is commonly adopted, so that both $\mathrm{T}$ and $\mathrm{R}$ modes can achieve maximum light efficiency and well matched gamma curves [1]-[3].

Moreover, to achieve a good dark state for the $\mathrm{T}$ and $\mathrm{R}$ modes simultaneously, several methods have been developed. A common approach is to use circular polarizers in both $\mathrm{T}$ and $\mathrm{R}$ regions [4], but this increases cost. Another method is to use patterned $\lambda / 4$ retarder only in the $\mathrm{R}$ region. So only light in $\mathrm{R}$ region experiences an additional $\lambda / 2$ phase change when traversing the LC layer twice. This $\lambda / 2$ phase change rotates the linearly polarized light $90^{\circ}$ before exiting the analyzer, and makes a single polarizer work as if two crossed linear polarizers [5], [6]. This patterned retarder requires a complicated fabrication processes and increases cost as well. As the nano-imprinting technology advances, nanowire grid polarizers could be used in TR-LCDs, which not only achieves a common dark state for $\mathrm{T}$ and $\mathrm{R}$ regions, but also recycles the light. However, the cost for this approach could be even higher [7].

In this paper, we propose a simple TR-LCD for mobile displays using double-cell-gap twisted nematic (TN) [8] for the $\mathrm{T}$ region and mixed twisted-nematic (MTN) [9] for the R region. By optimizing the orientation angles of optical components, LC twist angle, and phase retardation of the uniaxial positive A-film, the maximum light efficiency of $\mathrm{T}$ and $\mathrm{R}$ regions

Manuscript received December 22, 2008; revised March 07, 2009. Current version published July 29, 2009. This work was supported by Chi-Mei Optoelectronics Corporation (Taiwan).

The authors are with the CREOL, The College of Optics and Photonics, University of Central Florida, Orlando, FL 32816 USA (e-mail: \{yanli@ mail.ucf. edu; swu@mail.ucf.edu).

Color versions of one or more of the figures in this paper are available online at http://ieeexplore.ieee.org.

Digital Object Identifier 10.1109/JDT.2009.2021777

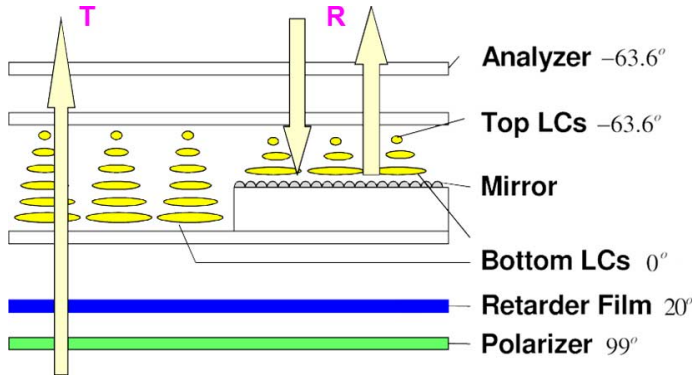

Fig. 1. Device structure of the normally black double-cell-gap TR-LCD. All the angles are referred to the horizontal axis.

using a $+\Delta \varepsilon$ LC achieves $87 \%$ and $96 \%$, respectively. Moreover, the voltage-dependent transmittance (VT) and voltage-dependent reflectance (VR) curves match very well, which enables a single gamma curve driving. The operating voltage is only $3.3 V_{\mathrm{rms}}$ which is desirable for low power mobile displays. Besides good optical performance, in this design, no patterned polarizer [3] or retarder [5], [6], or complicated photo-alignment process are required. The fabrication process of our design is fairly simple and the cost is low, making it suitable for mobile displays.

\section{Device Configuration}

Fig. 1 shows the device structure of our TR-LCD. Each pixel of the cell is divided into a $\mathrm{T}$ region and $\mathrm{R}$ region. The cell gaps of the two regions are different, but the LCs share the same twist angles in both regions: bottom rubbing angle is parallel to the $x$-axis and top rubbing angle is $63.6^{\circ}$. A same rubbing angle treatment enables a much simpler fabrication process. Although the $63.6^{\circ} \mathrm{MTN}$ cell was published years ago [10], it was under either parallel or crossed polarizer configuration. In our study, the two polarizers employed are neither parallel nor crossed, as shown in Fig. 1.

In the $\mathrm{R}$ region, light passes the analyzer and $\mathrm{LC}$ layer, and then is reflected back by the bumpy reflector. Without any additional compensation film here, it achieves a good dark state at $\lambda=550 \mathrm{~nm}$, when the analyzer and LCs are optimized as shown in Fig. 1 with $\mathrm{d} \Delta \mathrm{n}$ of the LC layer is equal to $201.5 \mathrm{~nm}$. After getting a good dark state for $\mathrm{R}$ region, we keep the analyzer and $\mathrm{LC}$ twist angle the same for $\mathrm{T}$ region. Then we adjust the $\mathrm{d} \Delta \mathrm{n}$ of the LC, the orientation and phase retardation of the compensation film below the LC layer, and the orientation of the polarizer, until T region also gets a good dark state. The final optimized orientation of the components is shown in Fig. 1, and the optimal $\mathrm{d} \Delta \mathrm{n}$ value is $416 \mathrm{~nm}$ for the $\mathrm{LC}$ in T region and is $441 \mathrm{~nm}$ for the positive uniaxial A-plate.

When no voltage is applied, both $\mathrm{T}$ and $\mathrm{R}$ regions exhibit a good dark state. As the voltage exceeds the threshold, the LC directors gradually tilt up vertically in response to the vertical 


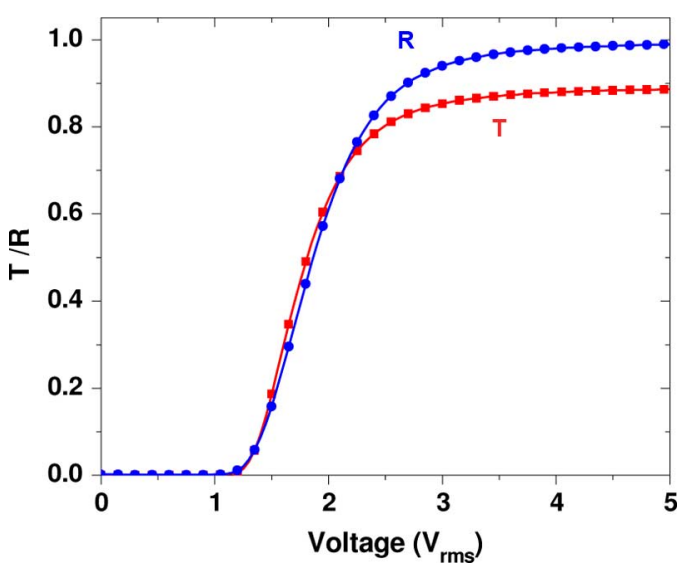

Fig. 2. Voltage dependent transmittance/reflectance of the $\mathrm{T}$ and $\mathrm{R}$ regions.

electric fields. For the on-state in the $\mathrm{R}$ region, it is as if the light passes through two parallel polarizers, resulting in a high the optical efficiency. In the T region, because of the phase retardation caused by the A-film, and the specific angles of these two polarizers, its optical efficiency is also quite high.

In a brief summary, our device has a relatively simple structure with the following features: 1) It does not require any patterned component, like patterned polarizer [3] or in-cell-retarder [5], [6]. 2) It only requires a double-cell-gap configuration, but the $\mathrm{LC}$ rubbing directions of the $\mathrm{T}$ and $\mathrm{R}$ regions are identical. The fabrication of double cell gap is no longer a problem, and has been widely used in commercial products, e.g., Sharp's dual-cell-gap ECB transflective displays [1]. 3) It uses only one uniaxial positive A-film. These features make our device attractive for ultra-thin profile portable displays.

\section{RESULTS}

To prove this device concept, we used Techwiz, a commercial 3D LCD software to simulate its electro-optic performances. The LC material we chose is TFT-graded materail MLC-6686. Its physical properties are as follows: $K_{11}=8.8 \mathrm{pN}, K_{33}=$ $14.6 \mathrm{pN}, \varepsilon / /=14.5, \varepsilon \perp=4.5, \gamma_{1}=102 \mathrm{mPas}, \Delta \mathrm{n}=0.0983$ at $\lambda=550 \mathrm{~nm}$. The cell gap for the $\mathrm{T}$ region is $4.25 \mu \mathrm{m}$ and for the $\mathrm{R}$ region is $2.05 \mu \mathrm{m}$. The positive Afilm has $\Delta \mathrm{n}=0.003$ at $\lambda=550 \mathrm{~nm}$ and $\mathrm{d}=147 \mu \mathrm{m}$. We also took into account the wavelength dispersion of the LC material, polarizers, and retarder film using the extended Cauchy equations [11], [12].

Fig. 2 shows the voltage-dependent transmittance and reflectance curves for the $\mathrm{T}$ and $\mathrm{R}$ regions. They have been normalized to the transmittance of two parallel polarizers, which is $\sim 34.33 \%$. The threshold voltage is found to be $\sim 1.1 V_{\mathrm{rms}}$.

At $3.3 V_{\text {rms }}$, the R region reaches $\sim 96 \%$ efficiency and the $\mathrm{T}$ region $\sim 87 \%$. The low operation voltage leads to low power consumption which is particularly attractive for mobile displays. Besides, the VT and VR curves match very well, indicating a single gamma driving can be employed for this display. The on-state efficiency is a little higher in $\mathrm{R}$ region than in $\mathrm{T}$ region. Because in the $\mathrm{R}$ region, it is just like passing through two parallel polarizers, and consequently, the optical efficiency is very high. But when we optimize the T region, we weighed

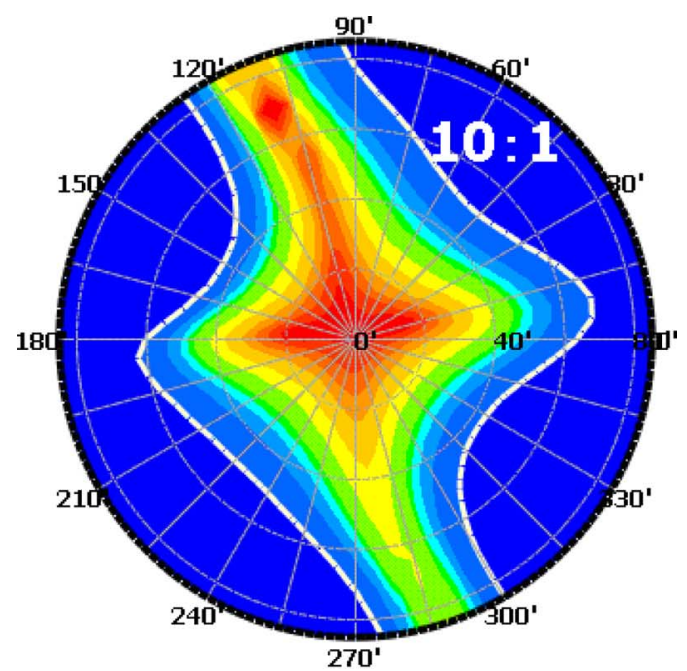

(a)

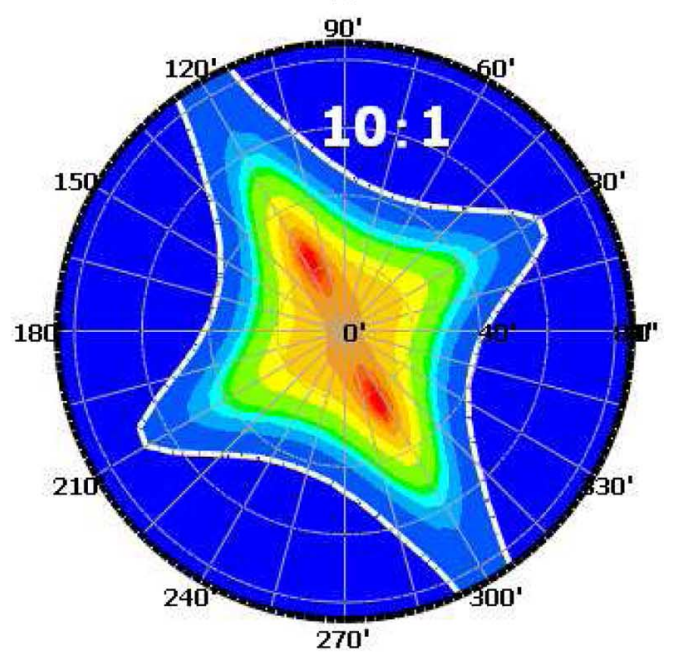

(b)

Fig. 3. Iso-contrast contour for (a) T region and (b) $\mathrm{R}$ region at $\lambda=550 \mathrm{~nm}$.

more in getting a perfect dark state than bright state. So, after light transmits through the polarizer, A-film, and LC cell in an on-state, its polarization direction is not exactly parallel to the transmission axis of the analyzer. As a result, a small portion of the light is absorbed by the analyzer, leading to a slightly lower optical efficiency than the $\mathrm{R}$ region.

We also calculated the response times of the $\mathrm{T}$ and $\mathrm{R}$ modes at $3.3 \mathrm{~V}_{\text {rms }}$. Results are listed as follows: For T mode, the rise time is $7.2 \mathrm{~ms}$ and decay time is $16.8 \mathrm{~ms}$. For $\mathrm{R}$ mode, the rise time is $2.9 \mathrm{~ms}$ and decay time is $4.2 \mathrm{~ms}$. The faster response time of $\mathrm{R}$ mode is due to its thinner cell gap.

Fig. 3(a) and (b) depicts the iso-contrast contour of the $\mathrm{T}$ and $\mathrm{R}$ modes, respectively. At normal incidence, the contrast ratio is higher than 4000:1 for the T region and higher than 500:1 for the $\mathrm{R}$ region. For the $\mathrm{T}$ region, the 10:1 iso-contrast contour is over $\sim 45^{\circ}$ viewing cone and for the $\mathrm{R}$ region it is $\sim 40^{\circ}$.

These results are typical for such a simple TN/MTN structure using only one A-film. Although the viewing angle is not very wide because only a single-domain LC structure and one compensation film are employed, it is still acceptable for small-panel mobile displays, such as an iPod. To widen the viewing angle for 


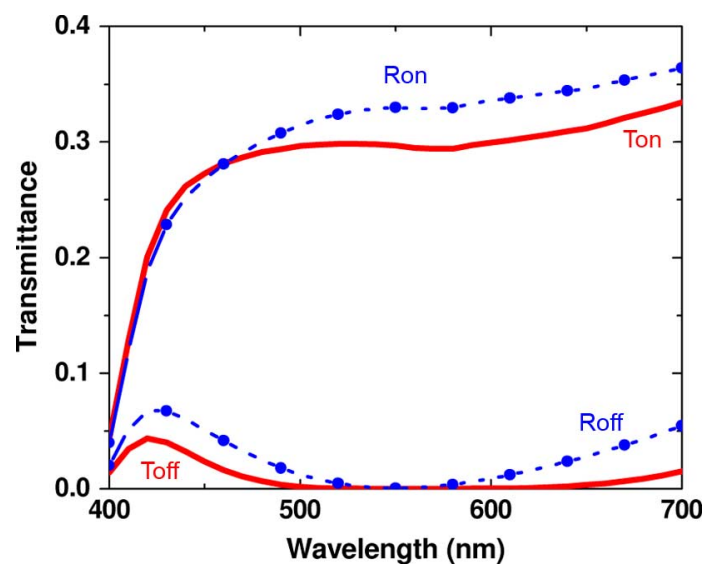

Fig. 4. Wavelength dependent transmittance and reflectance of the TR-LCD. $V_{\text {on }}=3.3 V_{\text {rms }}$ and $V_{\text {off }}=0$.

larger display panels, the Fuji wide-view discotic phase compensation film [13] could be used.

A general concern of this normally black TN/MTN mode is its dark state and contrast ratio sensitivity to cell gap, wavelength and angular variations. Therefore, we also evaluated the TR-LCD's performance over the entire visible spectrum and results are shown in Fig. 4. At $\lambda=550 \mathrm{~nm}$, the dark state for both $\mathrm{T}$ and $\mathrm{R}$ modes is very good, and the on-state efficiency is very high. However, as the wavelength departs from $550 \mathrm{~nm}$, the dark state light leakage gradually increases. This is more noticeable for the $\mathrm{R}$ mode than the $\mathrm{T}$ mode. We also evaluate the contrast ratio over entire visible wavelengths, using the following equation:

$$
\text { Contrast }=\frac{0.6 G_{1}+0.3 R_{1}+0.1 B_{1}}{0.6 G_{2}+0.3 R_{2}+0.1 B_{2}}
$$

where $R_{1}, G_{1}, B_{1}$ represent the on-state, while $R_{2}, G_{2}, B_{2}$ the off-state transmittance at red $(650 \mathrm{~nm})$, green $(550 \mathrm{~nm})$, and blue $(450 \mathrm{~nm})$ lights, respectively. The weights for R, G, B lights are roughly estimated from the human eye response function [14]. From our calculation, the overall contrast ratio for $\mathrm{T}$ region is $88: 1$, and for $\mathrm{R}$ region is $15: 1$. In a TR-LCD, the $\mathrm{R}$ mode is used mainly under bright ambient. Thus, the $\mathrm{R}$ mode performance is less stringent.

Next, we investigate the dark state tolerance of the TR-LCD to the $\mathrm{d} \Delta \mathrm{n}$ variation of the LC layer. The cell gap variation may originate from the manufacturing precision while $\Delta \mathrm{n}$ variation may come from the outdoor temperature change. Our simulation results are plotted in Fig. 5 for both $\mathrm{T}$ and $\mathrm{R}$ modes. From Fig. 5, as $\mathrm{d} \Delta \mathrm{n}$ departs from the optimal value (i.e., error $=0$ ), contrast ratio decreases gradually. At the optimal value, $T$ region could get a contrast ratio (CR) higher than 4000:1, and $\mathrm{R}$ region, more than 500:1. But even at $\pm 5 \%$ error, $\mathrm{T}$ region still maintains a $\mathrm{CR}>200: 1$, while $\mathrm{R}$ region $\mathrm{CR}>100: 1$ at normal incidence. To our surprise, the proposed TR-LCD is not too sensitive to the $\mathrm{d} \Delta \mathrm{n}$ fluctuation of the LC layer.

Finally, we examined its tolerance to angular variation. Since in industry, the angular control error of the optical components is usually within $\pm 1 \sim 2^{\circ}$, we let the analyzer, retardation film and polarizer deviate $\pm 2^{\circ}$ from their optimal orientations. The

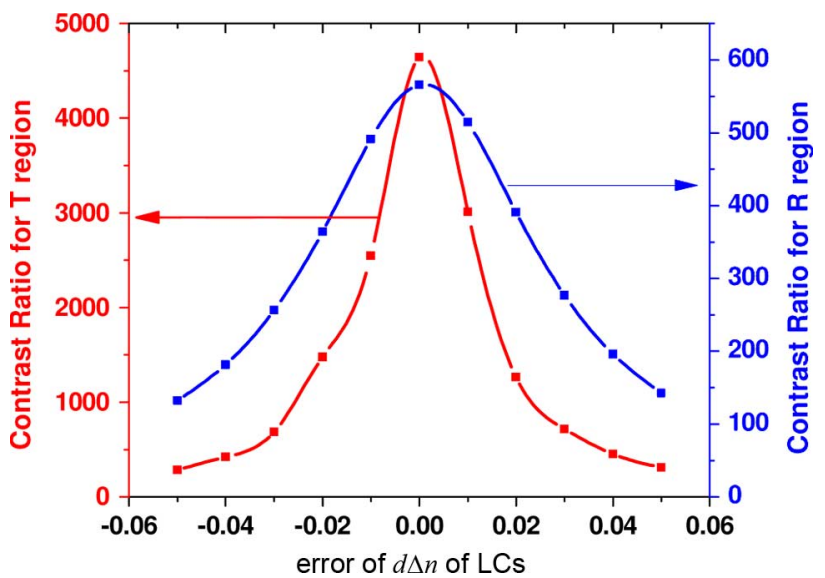

Fig. 5. Tolerance analyses of the TR-LCD. Here, $d \Delta \mathbf{n}$ of the LC departs from the optimal values by $\pm 5 \%$.

TABLE I

ANGUlar TOLERANCE OF THE CONTRAST RATIO.

$\begin{array}{llllllll}\begin{array}{l}\text { analyzer } \\ \left({ }^{\circ}\right)\end{array} & -63.6 & -61.6 & -65.6 & -63.6 & -63.6 & -63.6 & -61.6 \\ \begin{array}{l}\text { retarder } \\ \left({ }^{\circ}\right)\end{array} & 20 & 20 & 20 & 22 & 18 & 20 & 18 \\ \begin{array}{l}\text { polarizer } \\ \left(^{\circ}\right)\end{array} & 99 & 99 & 99 & 99 & 99 & 101 & 101 \\ \begin{array}{l}\text { contrast } \\ \text { T-mode } \\ \text { contrast }\end{array} & 4654 & 637 & 597 & 1242 & 2434 & 1173 & 259 \\ \text { R-mode } & 566 & 97 & 388 & - & - & - & -\end{array}$

results are shown in Table I. For the T region, even the worst case (the last column), the contrast ratio is still more than 200:1. For the $\mathrm{R}$ region, when we rotate the analyzer $\pm 2^{\circ}$ from the optimal position, the contrast ratio drops to $97: 1$ and $388: 1$, respectively. Therefore, the contrast ratio remains high even when optical components deviate from their optimal positions. On the other hand, we also examine the VT and VR curves and find that they hardly change in response to the orientation variation.

\section{CONCLUSION}

A simple TR-LCD with dual cell gaps is proposed. It exhibits some attractive features for mobile applications: 1) A simple device structure which implies to low cost and thin profile; 2) a low operation voltage at $\sim 3.3 V_{\text {rms }}$ resulting in a low power consumption and a long battery life; and 3) high transmittance and reflectance and a good match between VT and VR curves, which enables adequate for small-sized mobile displays. Therefore, this TR-LCD should be useful for low cost and thin profile mobile displays.

\section{REFERENCES}

[1] M. Okamoto, H. Hiraki, and S. Mitsui, "Liquid crystal display," U.S. 6281952, Aug. 28, 2001

[2] X. Zhu, Z. Ge, T. X. Wu, and S. T. Wu, "Transflective liquid crystal displays," J. Display Technol., vol. 1, no. 1, pp. 15-29, Sep. 2005

[3] H. Y. Mak and V. G. Chigrinov, "Transflective double cell TN liquid crystal display," in SID Symp. Dig., 2003, vol. 34, pp. 156-159.

[4] Z. Ge, R. Lu, T. X. Wu, and S. T. Wu, "Extraordinarily wide-view circular polarizers for liquid crystal displays," Opt. Express, vol. 16, pp. 3120-3129, 2008.

[5] J. B. Park, H. Y. Kim, Y. H. Jeong, S. Y. Kim, and Y. J. Lim, "Novel transflective display with fringe-field switching mode," Jpn. J. Appl. Phys., vol. 44, pt. 1, pp. 7524-7527, 2005. 
[6] G. S. Lee, J. C. Kim, T. H. Yoon, Y. S. Kim, W. S. Kang, and S. II. Park, "Design of wide-viewing-angle transflective IPS LCD," in Proc. 26th Int. Display Res. Conf., 2006, p. 75.

[7] Z. Ge and S. T. Wu, "Nano-wire grid polarizer for energy efficient and wide-view liquid crystal displays," Appl. Phys. Lett., vol. 93, p. 121104 , 2008.

[8] M. Schadt and W. Helfrich, "Voltage-dependent optical activity of a twisted nematic liquid crystal," Appl. Phys. Lett., vol. 18, pp. 127-128, Feb. 1971.

[9] S. T. Wu and C. S. Wu, "Mixed-mode twisted nematic liquid crystal cells for reflective displays," Appl. Phys. Lett., vol. 68, pp. 1455-1457, Mar. 1996.

[10] T. Sonehara and O. Okumura, "A new twisted nematic ECB mode for a reflective light valve," Proc. SID, vol. 31, pp. 145-148, 1990.

[11] S. T. Wu, C. S. Wu, M. Warengham, and M. Ismaili, "Refractive index dispersion of liquid crystals," Opt. Eng., vol. 32, pp. 1775-1780, Aug. 1993.

[12] J. Li and S. T. Wu, "Extended Cauchy equations for the refractive indices of liquid crystals," J. Appl. Phys., vol. 95, pp. 896-901, Feb. 2004.

[13] H. Mori, "The wide-view film for enhancing the field of view of LCDs," J. Display Technol., vol. 1, no. 2, pp. 179-186, Dec. 2005.

[14] G. Wyszecki and W. S. Stiles, Color Science. Hoboken, NJ: Wiley, 1982.

Yan Li received the M.S. degree in optical engineering from Zhejiang University (ZJU), Hangzhou, China, in 2007, and is currently in her second year working toward the Ph.D. degree at the College of Optics and Photonics, University of Central Florida, Orlando.

Her current research interests include energy efficient and fast response LCDs, and transflective LCDs.
Zhibing Ge received the B.S. degree in electrical engineering from Zhejiang University, Hangzhou, China, in 2002, and the M.S. and Ph.D. degrees in electrical engineering from University of Central Florida (UCF), Orlando, in 2004 and 2007, respectively.

Since 2008, he has been with the College of Optics and Photonics at University of Central Florida as a research scientist. His research interests include novel liquid crystal displays and laser beam steering technologies. He has published 1 book chapter, over 30 journal papers, and 12 issued or pending U.S. patents in related area.

Dr. Ge is a recipient of the 2008 Otto Lehmann Award.

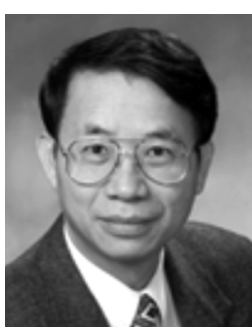

Shin-Tson Wu (M'98-SM'99-F'04) received the B.S. degree in physics from National Taiwan University, and the Ph.D. degree from the University of Southern California, Los Angeles.

$\mathrm{He}$ is a PREP professor at College of Optics and Photonics, University of Central Florida (UCF). His studies at UCF concentrate in liquid crystal displays, liquid crystal materials, optical communications, photonic crystal fibers, and bio-photonics. Prior to joining UCF in 2001, he worked at Hughes Research Laboratories, Malibu, CA, for 18 years. He has co-authored 5 books: Introduction to Flat Panel Displays (Wiley, 2008, with J. H. Lee and D. N. Liu), Fundamentals of Liquid Crystal Devices (Wiley, 2006, with D. K. Yang); Introduction to Microdisplays (Wiley, 2006, with D. Armitage and I. Underwood), Reflective Liquid Crystal Displays (Wiley, 2001, with D. K. Yang) and Optics and Nonlinear Optics of Liquid Crystals (World Scientific, 1993, with I. C. Khoo), 6 book chapters, over 300 journal publications, and more than 58 issued patents.

Dr. Wu is a recipient of SPIE G. G. Stokes award and SID Jan Rajchman prize. $\mathrm{He}$ is a Fellow of the Society of Information Display (SID), Optical Society of America (OSA), and SPIE. 